\title{
DEMAND SIDE MANAGEMENT AS A SUPPORT TO DISTRIBUTED GENERATION IN ACTIVE NETWORKS
}

\author{
G. CELLI, E. GHIANI, S. MOCCI, F. PILO, and E. PAZZOLA \\ Department of Electrical and Electronic Engineering - University of Cagliari - Italy \\ celli@diee.unica.it, eghiani@diee.unica.it, susanna.mocci@diee.unica.it, pilo@diee.unica.it
}

\section{SUMMARY}

In the last decade, it has been witnessed an increasing allocation of Distributed Generation (DG) in the $M V$ distribution networks, due to technology improvement, energy market liberalization, and environmental issues. Every year, Distribution Network Operators (DNOs) receive several requests for installations of new generators in their networks. This situation will likely imply a revolution of the distribution networks, actually characterized by radial structure and passive operation, in order to exalt the benefits and reduce the drawbacks that DG can introduce. Therefore, it is predictable in the near future the implementation of an active management of the distribution networks. Active networks in the distribution of the future will require a large use of ICT and automatic control systems to control loads and generators, with the aim of allowing the participation of DERs to the energy and services market and of maintaining a sufficient level of system reliability. Into this scenario, DG and Demand Side Management (DSM) actions should become options that distributors have to consider in order to solve electric supply problems, as requested by the more recent EU directives. In the paper it has been analysed how DSM policies can be a valid opportunity to facilitate the development of DG in a given distribution system and which economical benefits the utilities can derive by the complementary employ of both these distributed resources. Simulation studies have been performed on real Italian distribution networks, showing the effects of DSM actions on the growth of DG in the distribution system and on the technical and economic benefits, they permit to realize.

\section{INTRODUCTION}

The need for more flexible and secure electric systems, the changing in the regulatory and economic scenarios, the importance of harnessing energy savings and minimizing environmental impacts have been providing the impetus for an intensive integration of Distributed Generation (DG) in the distribution system. Unfortunately, the distribution network had not been designed to have significant quantities of generation connected and for this reason the distribution operators are used to apply the "connect and forget” rule. Such rule implies that only those generators that do not alter the normal functioning of a network may be allowed to be connected. Thus, the DG effect on the network is assessed considering the two extreme situations of no load/maximum generation and maximum load/no generation. If all technical constraints are complied with, the "connect and forget" rule is respected and DG can be connected, otherwise the most obvious solution is the connection to a higher voltage. Generally, the higher is the voltage level the higher are the connection costs and, consequently, the lower is the profitability of the investments. This rule poses a strong barrier to the integration of DG in general and to wind generation in rural networks in particular. Finally, it should be recognised that in some case the "connect and forget" rule may be unfair because penalises the last generator asking to be connected and does not consider the effect of the others. The passive operation of the distribution systems causes technical and economic barriers that still limit the use of DG and RES (Renewable Energy Sources) and cannot be tolerated in the EU, where stringent requirements on the reduction of the $\mathrm{CO}_{2}$ pollution have been imposed [1].

Moreover, according to the EU directive EU/54/2003 DG and DSM should be considered possible valid planning alternatives to face distribution expansion problems. Thus, deterministic network studies as well as the passive operation of the distribution system are probably destined to be abandoned [2]-[4].

Active network, in opposition to traditional passive distribution systems, which have only to supply end users, means a distribution system where customers, power producers and DSO actively interact to sell or purchase energy and to manage and operate the network [4]. Thus, the distribution of the future will be no longer a passive termination of the transmission grid. The concept of active network involves the adoption of dynamic voltage systems, the dispatching of active and reactive powers, the resorting to responsive loads and DSM policies, the massive use of the most advanced information, communication and automation technologies, and finally a whole liberalized energy market for customers and producers. Such a drastic and revolutionary change will not suddenly take place due to the investments it requires but, more likely, it will be a gradual step-by-step procedure driven by market and political decisions.

The first level of an active network may be based on the generation curtailment during low load conditions that cause non-tolerable overvoltages. The main idea behind the generation curtailment is that a power producer may find economically convenient to be cut off in some circumstances if he can install a bigger power generator and sell more energy for the majority of the time. This is certainly the simplest arrangement of an active network but it may be useful especially in case of wind generation connected in rural areas. The paper aims at comparing the effect of the generation curtailment with a simple DSM policy, essentially based on the idea of moving some energy consumption from the peak hours to off-load ones by controlling some loads with storage features. In this way, much more generation can be tolerated in the network and overvoltages may be reduced 
within the limit imposed by DSO. In particular, the greater attention has been paid to a parametric analysis of the DSM cost that can allow better results in comparison to the generation curtailment approach.

\section{VOLTAGE RISE EFFECT}

The voltage rise caused by DG is a well known effect and can be illustrated using the simple circuit shown in Fig. 1. This figure represents the basic features of a distribution system into which a distributed generator, $\mathrm{G}$, is connected at the MV level. The generator is connected to the distribution system through a weak network (high equivalent impedance $\mathrm{Z}$ at the connection point) and an on-load tap changing transformers. The scheme is completed with a load and a reactive power compensator. The voltage at busbar 2 is expressed by (1), where $P_{G}$ and $Q_{G}$ are the generated active and reactive power, $P_{L}$ and $Q_{L}$ are the active and reactive power drawn by the load, $Q_{C}$ is the reactive power required by the reactive power compensator. $\triangle P_{D S M}$ is the portion of the total load that can be moved from the peak hours to the off-peak hours.

$$
V_{2} \approx V_{1}+R \cdot\left(P_{G}-P_{L} \pm \Delta P_{D S M}\right) \pm X \cdot\left( \pm Q_{G}-Q_{L} \pm Q_{C}\right)
$$

This simple equation can be used to qualitatively analyse the relationship between the voltage at busbar 2 and the amount of generation that can be connected to the distribution network, as well as the impact of alternative control actions. As indicated earlier, the general practice in distribution networks is to limit the capacity of the connected DG based on the extreme conditions of minimum load $\left(P_{L}=0, Q_{L}=0\right.$ and $\left.\Delta P_{D S M}=0\right)$ and maximum generation $\left(P_{G}=P_{G M A X}\right)$. Under such hypothesis the maximum level of generation that can be connected to the network (with no reactive power compensation and generator at unitary power factor) is expressed by Eq. (2)

$$
P_{G M A X} \leq \frac{V_{2 M a x}-V_{1}}{R}
$$

where $V_{2 M a x}$ represents the maximum voltage rise admitted at the busbar 2. It is trivial to observe that, by applying the rule, only few generators can be connected without reinforcing the network. Of course, the reactive power regulation can help to increase the maximum allowable penetration of DG as shown in [4] but, for the sake of simplicity, in this paper only active power has been considered.

The probability of occurrence of the extreme situation in (2) is generally quite low and for this reason in [4], [5] it has been supposed to actively managing the network so that generation is curtailed during inadmissible over-voltages at busbar 2 .

The probability of the coincidence of minimum load demand and maximum generation will determine the total annual

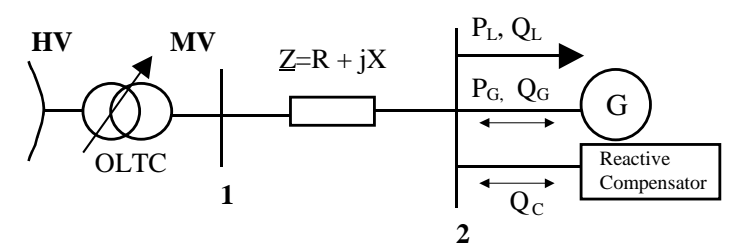

Fig. 1 - The simplified scheme for modelling the voltage rise due to DG energy curtailed. As the price of electricity is primarily driven by load demand, and generation curtailment occurs typically during periods of low load, the value of this energy curtailed is likely to be relatively low.

In this paper, a similar approach has been followed but, in order to make a comparison, the effect of a DSM action able to reduce the peak load by increasing the energy demand during the low load conditions has been investigated. The Eq. (3) shows the potential effect on the $P_{G \max }$ of the $\Delta P_{D S M}$.

$$
P_{G M A X} \leq \frac{V_{2 M a x}-V_{1}}{R}+\frac{\Delta P_{D S M}}{R}
$$

Eq. (3) shows a linear relationship between the portion of loads involved in the DSM program and the increasing of the maximum allowable level of DG. In this particular case, some loads can be controlled by the DSO and agree to be connected when necessary (e.g. loads with storage features) or some other loads are moved to change their normal behaviour and convinced to use more energy during off-peak hours. Naturally, it is very difficult to economically justify a DSM with the final goal of accommodating more DG in the distribution system but, if a DSM policy is going to be adopted for various reasons, then it may be advantageously used also for accommodating more DG [5]. In this case, the DG investor can be motivated to sustain the DSM action because this action can allow increasing its annual revenue. In the paper it has been assumed that the DG owner agrees to pay the energy corresponding to the $\Delta P_{D S M}$, which is necessary to accommodate the power produced. Considering that voltage rise problems generally occur during low cost hours, some hypothesises have been made on the cost of the DSM service. In other words, DG owners accept to pay a fee for the active network service (in this case the active network service is constituted by the action on loads) but they can have a major income by selling more energy during the peak hours.

\section{DEMAND SIDE MANAGMENT}

The possibility of an electric load to participate in an offer implying energy reduction - demand offer - depends on the ability of the user to switch off or reduce partially the load demand while maintaining end-use characteristics of such a load - comfort or processes - in certain levels [6].

Several factors are relevant to classify load capability for this purpose:

- final service supplied by the electric load (thermal, mechanical, electronic);

- $\quad$ storage capacity;

- rate of load switching;

- load dispatch facility.

The first one refers to the energy conversion that is being performed in the end-user device, independently of the nature of the individual elements that may compose the load (for example, an air conditioning set is providing cool air through the conversion of electrical energy into mechanical energy in a motor that powers a compressor). Electricity is more valuable than other energy sources due to its flexibility for energy conversion but, for a few end uses, other forms of primary energy could compete because of their higher 
efficiency. Dual heating or cooling appliances are candidates to support dual energy systems (electricity and gas) and that has to be taken into account to perform demand offers and bids.

The storage capacity is related to the ability of the whole process to store some kind of energy (electrical, thermal, or in the form of hydrogen) or some other "manufactured products" that can be used in any other time. The storage capability should allow the electric consumption process to be rescheduled over a specified time span. The energy storage capacity can be internal to the load (i.e., the load has a device or system that stores the excess of electricity with batteries or other, or the converted energy as, for example, in the form of ice for cool storage or heated bricks for heat storage). Sometimes the environment of the load has some kind of energy storage availability (or external storage) as, for example, the one provided by the storage capacity due to the specific heat of the air, walls, furniture, etc. located in an office or dwelling in an air conditioning/heating load. The internal storage can be either partial or total according to the global energy provided by the electric load in a load cycle. The third factor is related to the load demand behaviour. The load pattern of consumption, according to the electrical device nature, can be discrete or continuous. A continuous demand to accomplish the service provided by the load may result in a continuous electricity consumption (this is the case of lighting loads or computers) or in a discontinuous consumption (for example, in a refrigerator or in most air conditioning devices where the operating state is thermostat controlled). In both cases, the nature of the load demand is driven by the random nature of the load usage, which introduces an additional discrete type of uncertainty to the electrical behaviour of the load. Thus, the rate of load switching conditions the frequency and the amplitude in which the demand of an individual load or an aggregation of them can be modified. It is interesting to note that in this type of load, the load supply switching could not result in electric energy increase or reduction as the load may be supplying the required service from stored resources.

Finally, the load dispatch capability refers to the availability of communication/control mechanisms so that this load can be managed both in a local or remote fashion, and with the necessary response time to allow an effective control policy at an affordable economic cost.

The control performed upon the loads can be local, affecting only the load element (i.e., a local thermostat), or remote by some other control system. This control can involve a total load switch off (with complete loss of the service provided by the load when interrupted), or partial, where the electric load supply is bounded by cycling or voltage modulation policies (resulting in a partial loss of the load service). In order to find the suitability of the electrical loads to participate in demand reduction or modulation programs, the effect of the possible control actions in the power supply has to be evaluated and modelled. The most suitable way to account for all of the different modelling requirements to be considered in the electrical demand bids generation is through physically based load models that offers the possibility to model the joint behaviour of the electrical devices forming the load as well as all of the associated physical devices.
In the paper the load control has been used to solve voltage rise problems. Normally, load control is traditionally used by electricity utilities for peak lopping purposes. Using load control to limit distribution system voltage rise is in contrast to peak lopping since the requirement is to fill in demand minima during periods of high generation. Thus, when feeder voltages are at their limit, additional load is controlled on to reduce voltage. The quality of the load control policy depends on the size of the load group that can be switched on to follow the generation (e.g. wind) variability. The faster and capillary is the load control adopted, the more effective is the voltage regulation. This requires a better control and communication system but is much more energy efficient. The loads with storage capabilities are the most appropriate for control such as thermal storage heaters and hotwater storage systems. Controlling such loads "on" creates virtually no consumer inconvenience and allows the "surplus" energy to be used usefully in a thermal state later rather than discarded as with generation constraint for example. It should be recognised that this is a very important point especially if it is observed that the energy for the same storage loads in the peak hours could have to be produced with traditional and less environmental compatible generators.

\section{RESULTS AND DISCUSSIONS}

In order to study the performance of the DSM actions, some tests have been performed on the small test system depicted in Fig. 2. In order to be able to make a comparison with the results presented in [4], the generation curtailment has been also applied to the same case study. To simplify the model, only two energy prices have been considered, one for the peak hours and one for the off-peak hours (according to Italian data, the prices have been assumed equal to 115.31 $€ / \mathrm{MWh}$ and $35.52 € / \mathrm{MWh})$. The loads have been grouped into three lumped loads drowning 31.5 MW, 13.1 MW, and 3.3 MW from busses 3, 4, and 5 respectively (Fig. 2) during the off-peak hours; all loads have 0.9 lagging power factor. The wind generator has been assumed equivalent to a constant generator producing the rated power for 1860 hours/year (the proportion between the peak hours and the off-peak hours has been set equal to the ratio 690/1170). During the observed period, the number of hours with an intolerable voltage rise is necessarily related to the rated power of the wind generation (see Table I, row 2) and naturally, it increases with the rated power. The same behaviour is also showed by the percentage of load participating to the DSM policy (see Table I, row 3), calculated with reference to the minimum of the power demand. As it can be easily seen, the network can accommodate only a $6 \mathrm{MW}$ generator without any other adjunctive action. More generation installed requires DSM actions for the hours indicated in Tab. I. The same result can also be achieved with the generation curtailment option.

Despite the number of hours of no production in the generation curtailment case [4] or the fee that should be paid by DG in the DSM case, the DG owner revenue increases thanks to the possibility of producing more energy during the peak hours, when the energy price rises to very high levels. In Fig. 2 a comparison between the two options is proposed. 


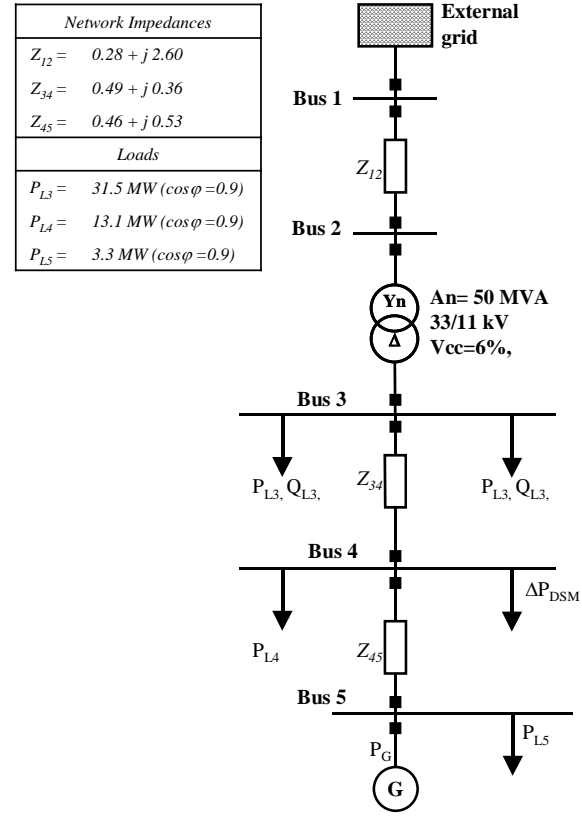

Fig. 2 - The test system used to show the effect of DSM on the maximum allowable DG penetration

Clearly, the benefit of the DSM depends on the fee paid by DG [7]. Whether the DSM fee is established equal to the energy price during the off-peak hours, the generation curtailment is slightly more advantageous, especially for the highest value of DG installed. If the cost of DSM was set equal to the difference, which has to be recognised to those customers that change their load curve to increase the energy demand during the off-peak hours, the DG owner revenue is greater than in the curtailment case. From this simple example, it is evident that the generation curtailment is more convenient than the load control. Furthermore, whether capital costs are taken into account, the comparison is much

Table I - Relationship between the DG power installed and the number of hours requiring DSM or Generation Curtailment

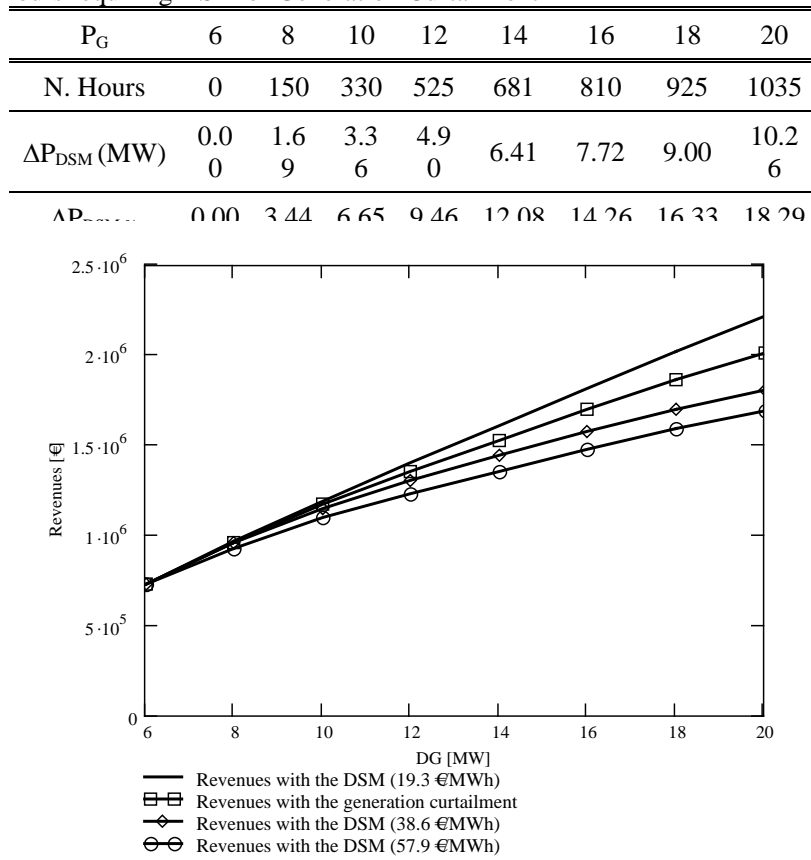

Fig. 3 - Annual revenues with the DSM or the generation curtailment as a function of the DG power installed less favourable to the load control, because it is more expensive than the other "dynamic" methods such as generation curtailment or constraining and power factor control [5].

\section{CONCLUSIONS}

Nowadays, the need for more flexible electric systems and the importance of harnessing energy savings and minimizing environmental impacts are changing the structure of the distribution system. Nevertheless, the current distribution system is essentially passive and no voltage regulation is adopted. This causes an important barrier to the integration of wind based DG due to the risk of intolerable voltage rises during low load hours. Actually, the risk is not really as high as it can appear by applying a deterministic approach and the resort to a better suited probabilistic approach is mandatory not to hide the real benefits of DG. Anyway, several actions are available to face voltage rise concerns without limiting the size of the DG installed. In the paper the possibility of adopting a DSM policy based on the switching of some storage loads is assessed. The preliminary tests performed have shown that the DSM action, depending on the active network cost paid by the DG owner, can give results comparable with the generation curtailment, even if, as first important remark, the generation curtailment is more convenient because generally it requires fewer investments. Nevertheless, DSM and load control are able to give the distribution system more benefits (e.g. they can be used to defer network investments due to the load peak and to exalt the benefits achievable from many small storage loads) and a fairer comparison should consider this aspect.

\section{REFERENCES}

[1] European white paper for a community strategy and action plan, "Energy for the future renewable sources of energy", in Proc. COM, Nov. 1997.

[2] G. Carpinelli, G. Celli, F. Pilo, and A. Russo, "Distributed Generation planning under uncertainty including power quality issues”, ETEP, vol. 13, no. 6, pp. 381-389, Nov./Dec. 2003.

[3] N. D. Hatziargyriou, T. S. Karakatsanis, and M. Papadopoulos, "Probabilistic load flow in distribution systems containing dispersed wind power generation,” IEEE Trans. Power Syst., vol. 8, pp. 159-165, Feb. 1993.

[4] S.N. Liew and G. Strbac, "Maximising penetration of wind generation in existing distribution networks", IEE Proc. Gener. Transm. Dist., Vol. 149, No 3, May 2002.

[5] N. C. Scott, D. J. Atkinson, J. E. Morrell, "Use of Load Control to Regulate Voltage on Distribution Networks with Embedded Generation”, IEEE Trans. on Power Systems, vol. 17, n², May 2002.

[6] C. Alvarez, A. Gabaldòn, A. Molina, “Assessment and Simulation of the Responsive Demand Potential in end-User Facilities: Application to a University Customer”, IEEE Trans. on Power Systems, vol. 19, n², May 2004.

[7] J. Mutale, G. Strbac, "A case study of active management of distribution networks with distributed generation in the UK”, deliverable 4.2 of the BUSMOD project, March 2004). 